\title{
Исследование механизмов токопрохождения в гетероструктуре $\mathrm{CdS} /$ por-Si/p-Si
}

\author{
(C) В.В. Трегулов ${ }^{1}$, В.Г. Литвинов ${ }^{2}$, А.В. Ермачихин ${ }^{2}$ \\ ${ }^{1}$ Рязанский государственный университет им. С.А. Есенина, \\ 390000 Рязань, Россия \\ ${ }^{2}$ Рязанский государственный радиотехнический университет, \\ 390005 Рязань, Россия \\ E-mail: trww@yandex.ru
}

(Получена 16 мая 2017 г. Принята к печати 22 ноября 2017 г.)

Исследована температурная зависимость прямых и обратных ветвей вольт-амперной характеристики, а также спектр фотоэдс полупроводниковой гетероструктуры $\mathrm{CdS} / p o r-\mathrm{Si} / p-\mathrm{Si}$. Установлено, что механизмы токопрохождения определяются генерационно-рекомбинационными процессами в области пространственного заряда гетероперехода $p o r-\mathrm{Si} / p-\mathrm{Si}$, туннелированием носителей заряда в пленке por-Si и моделью токов, ограниченных пространственным зарядом. Предложен упрощенный вариант зонной диаграммы исследуемой гетероструктуры.

DOI: $10.21883 /$ FTP.2018.07.46047.8648

\section{1. Введение}

Пористый кремний (por-Si) считается перспективным материалом для применения в современной оптоэлектронике. Формирование пленки por-Si на фронтальной поверхности традиционного фотоэлектрического преобразователя солнечной энергии позволяет существенно улучшить его характеристики за счет снижения потерь на отражение и пассивации дефектов [1]. Также на основе гетероперехода между por-Si и монокристаллическим кремнием могут быть созданы быстродействующие фотодиоды, способные регистрировать наносекундные световые импульсы [2].

В последнее время усилился интерес к структурам, в которых на поверхности por-Si сформированы полупроводниковые пленки, такие как $\mathrm{ZnO}$ [3], $\mathrm{CdS}$ [4,5] и др. Прежде всего это вызвано стремлением расширить спектральный диапазон фоточувствительности.

Данная статья посвящена исследованию гетероструктуры $\mathrm{CdS} /$ por-Si/p-Si. Пленки CdS могут успешно применяться при создании фотоэлектрических преобразователей солнечной энергии с поглощающим слоем на основе кремния. Согласно [6], эффективность фотоэлектрического преобразователя на основе гетероперехода $\mathrm{CdS} / p$-Si может достигать 24\%. Здесь пленка $\mathrm{CdS}$ играет роль оптического окна и существенно расширяет область спектральной чувствительности по сравнению с традиционным преобразователем на основе $p$-n-перехода [6]. Для формирования пленки $\mathrm{CdS}$ в исследованной в данной работе гетероструктуpe, использовался метод гидрохимического осаждения, который в литературе также называют методом химической ванны [7]. Главными достоинствами данного метода являются простота реализации и возможность получения достаточно качественных пленок $\mathrm{CdS}$ на подложках с большой площадью поверхности, пригодных для использования в качестве оконных слоев фотоэлектрических преобразователей солнечной энергии и оптических датчиков [7]. Также следует отметить, что слой por-Si может играть роль буфера [8], снижающего механические напряжения, возникающие между кремниевой подложкой и выращиваемой пленкой $\mathrm{CdS}$.

Несмотря на достаточно большое внимание к гетероструктурам $\mathrm{CdS} /$ por- $\mathrm{Si} / p$ - $\mathrm{Si}$, механизмы токопрохождения в них исследованы недостаточно. В то же время такие исследования актуальны, потому что особенности механизмов токопрохождения в значительной степени определяют основные характеристики полупроводниковых приборов.

Цель настоящей работы состоит в изучении механизмов токопрохождения в полупроводниковой структуре $\mathrm{CdS} /$ por $-\mathrm{Si} /$ p-Si на основе исследования температурной зависимости темновых вольт-амперных характеристик и спектров фотоэдс.

\section{2. Описание образцов и методики исследования}

Основой исследуемой структуры является кремниевая монокристаллическая подложка $p$-типа проводимости с ориентацией поверхности (100) и удельным сопротивлением 1 Ом · см. Пленка por-Si выращивалась на поверхности подложки методом анодного электрохимического травления в электролите, состоящем из смеси НF и $\mathrm{C}_{2} \mathrm{H}_{5} \mathrm{OH}$ в соотношении $1: 1$. Электрохимическое травление производилось в гальваностатическом режиме при плотности тока $18 \mathrm{~mA} / \mathrm{cm}^{2}$ в течение 10 мин. В качестве катода использовался спектрально-чистый графит. После выращивания пленки por-Si производилось травление поверхности образца в водном растворе НF (10\%) в течение 10 мин. В результате толщина пленки por-Si составила 2.2 мкм. 
Пленка $\mathrm{CdS}$ формировалась на поверхности слоя por-Si осаждением из водных растворов хлорида кадмия $\mathrm{CdCl}_{2}$ (концентрация $0.44 \mathrm{M}$ ) и тиомочевины $\mathrm{N}_{2} \mathrm{H}_{4} \mathrm{CS}$ (концентрация $0.22 \mathrm{M}$ ). Хлорид кадмия является источником ионов кадмия, тиомочевина - источником ионов серы для образования соединения $\mathrm{CdS}$. В качестве комплексообразователя использовался концентрированный водный раствор аммиака $\mathrm{NH}_{4} \mathrm{OH}$. Вначале к раствору $\mathrm{CdCl}_{2}$ добавлялся раствор аммиака до полного растворения выпавшего осадка, затем к полученному раствору добавлялся такой же объем водного раствора тиомочевины. Далее в раствор погружались подложки с предварительно сформированной пленкой por-Si, проводилось нагревание до $90^{\circ} \mathrm{C}$ и в течение 20 мин выращивалась пленка $\mathrm{CdS}$. Затем слой $\mathrm{CdS}$ со стороны $p$-Si полностью стравливался 30\%-м раствором $\mathrm{HCl}$. Образцы промывались дистиллированной водой и высушивались в сушильном шкафу. Пленка $\mathrm{CdS}$ имела $n$-тип проводимости, ее толщина составляла 1.8 мкм.

На заключительном этапе формировались омические контакты к подложке $p$-Si и пленке $\mathrm{CdS}$ посредством пайки индия.

Для исследования фотоэлектрических свойств измерялись спектры фотоэдс при температуре $300 \mathrm{~K}$ с помощью экспериментальной установки на основе монохроматора УМ-2, селективного вольтметра В3-38 и обтюратора с частотой вращения диска 7 Гц. Освещение исследуемой структуры производилось со стороны слоя $\mathrm{CdS}$, вдоль нормали к фронтальной поверхности.

C целью изучения механизмов токопрохождения проводились измерения вольт-амперных характеристик $(\mathrm{BAX})$ в прямом и обратном смещении в диапазоне температур 80-300 K с шагом $10 \mathrm{~K}$, без освещения поверхности исследуемого образца. Для измерений использовалась экспериментальная установка на базе гелиевого криостата замкнутого типа Janis CCS 400/204N и электрометра Keithley-6517B со встроенным источником постоянного напряжения. При измерении ВАХ прямое смещение исследуемой полупроводниковой структуры соответствует приложению отрицательного потенциала к контакту на поверхности слоя $\mathrm{CdS}$, положительного потенциала — к контакту на $p$-Si.

\section{3. Результаты эксперимента и их обсуждение}

Спектры нормированной фотоэдс $\left(U_{O C}\right)$ исследуемой структуры представлены на рис. 1. Для сравнения на рис. 1 приведен спектр для кремниевого фотоэлектрического преобразователя на основе $n^{+}-p$-перехода.

При освещении исследуемой полупроводниковой структуры со стороны слоя $\mathrm{CdS}$ на контакте к $\mathrm{CdS}$ появляется отрицательный потенциал, на контакте к $p$-Si - положительный. Длинноволновая граница спектра гетероструктуры $\mathrm{CdS} /$ por-Si/p-Si совпадает с краем поглощения монокристаллического кремния (рис. 1).

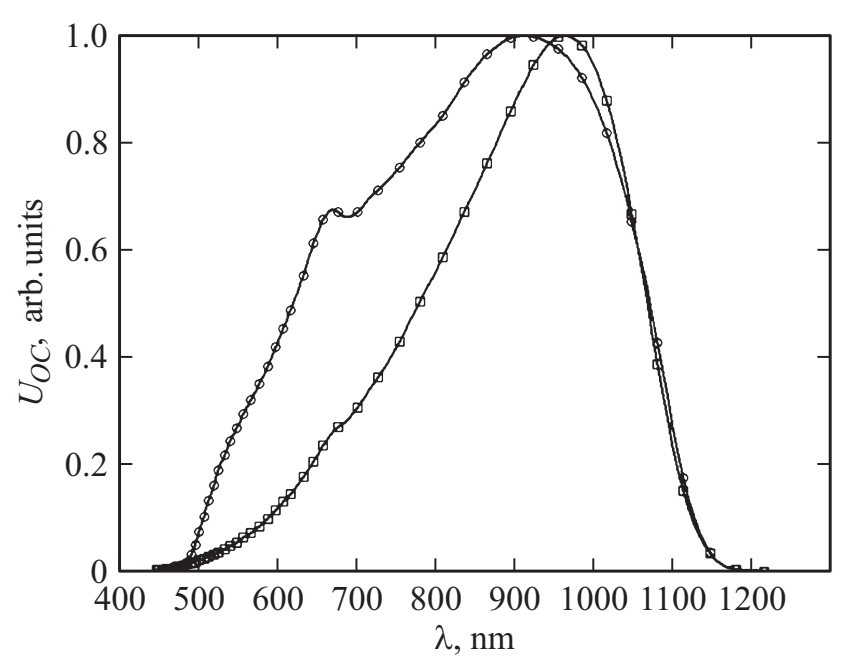

Рис. 1. Спектры фотоэдс гетероструктуры $\mathrm{CdS} /$ por- $\mathrm{Si} / p$-Si (o) и кремниевого фотоэлектрического преобразователя на основе $n^{+}-p$-перехода ( $\left.\square\right)$.

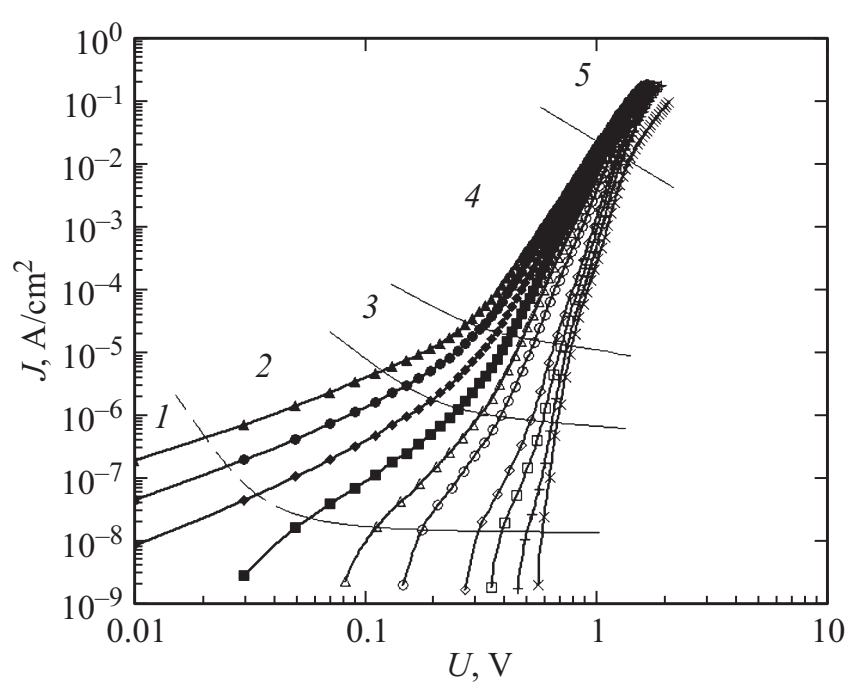

Рис. 2. Прямые ветви ВАХ при температуре $T, \mathrm{~K}: \times-80$, $+-110, \square-140, \diamond-160, \circ-200, \triangle-220, \mathbf{\square}-240, \bullet-$ $260, \bullet-280, \wedge-300$.

Это свидетельствует о преимущественном поглощении длинноволнового излучения в $p$-Si.

Прямые ветви ВАХ, измеренные в диапазоне абсолютных температур $(T) 80-300 \mathrm{~K}$, представлены на рис. 2 в виде зависимости плотности тока $J$ от приложенного постоянного напряжения смещения $U$ в двойном логарифмическом масштабе. Ветви ВАХ при обратном смещении, измеренные в том же диапазоне температур, представлены на рис. 3 в линейном масштабе. Из рис. 2 и 3 следует, что BAX структуры $\mathrm{CdS} /$ por $-\mathrm{Si} / p-\mathrm{Si}$ имеют выпрямляющий характер. Величина коэффициента выпрямления, определенная как отношение значений плотностей токов при $U=1.5 \mathrm{~B}$ для прямого и обратного 


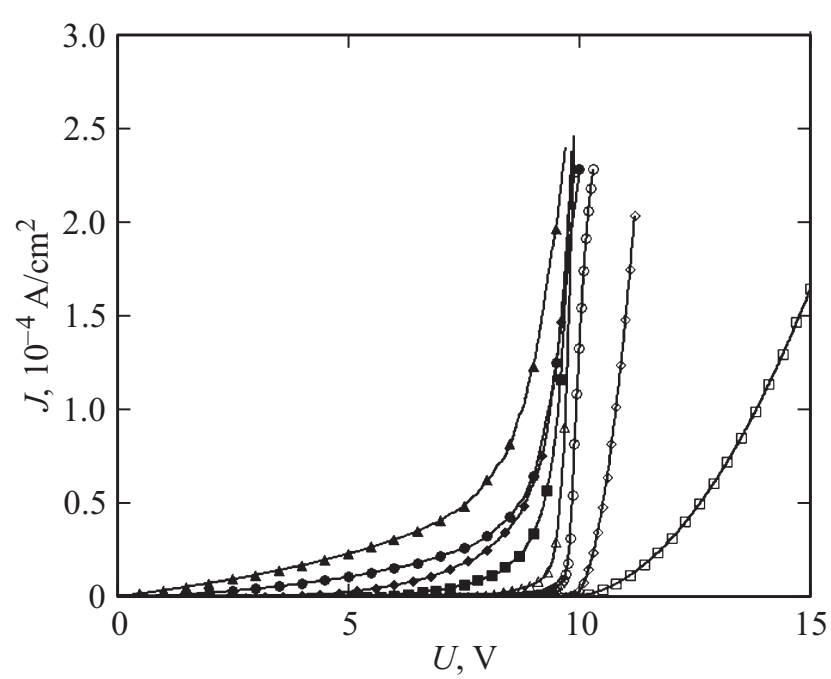

Рис. 3. Обратные ветви ВАХ при температуре $T, \mathrm{~K}: \square-80$, $\diamond-110, \circ-140, \triangle-160, \mathbf{\square}-200, \bullet-240, \bullet-280$, $\Delta-300$.

смещения, составила $1.41 \cdot 10^{6}$ при $T=80 \mathrm{~K}$ и $2.37 \cdot 10^{4}$ при $T=300 \mathrm{~K}$.

На прямых ветвях ВАХ (рис. 2) можно выделить 5 участков, которые приблизительно аппроксимируются отрезками прямых и описываются степенной зависимостью вида

$$
J \propto U^{m},
$$

где $m$ - показатель степени, характеризующий наклон соответствующего участка [9].

Для участка 1 прямых ветвей $\mathrm{BAX}$ (рис. 2) при $T=80-240 \mathrm{~K}$ величина $m>2$. Это может быть объяснено в рамках модели токов, ограниченных пространственным зарядом (ТОПЗ), влиянием на процессы токопрохождения ловушек с экспоненциальным распределением по энергии активации [9]. При $T=250-300 \mathrm{~K}$ участки 1 и 2 практически сливаются (рис. 2) и выполняется условие $1<m<2$. С точки зрения модели ТОПЗ это означает, что концентрация инжектированных носителей заряда становится сравнимой с концентрацией термически генерированных носителей [9].

Одновременно участок 2 при $T=80-300 \mathrm{~K}$ может быть представлен экспоненциальной зависимостью вида

$$
J \propto \exp \left(\frac{q U}{n k T}\right)
$$

где $q$ - элементарный заряд, $n$ - показатель неидеальности гетероперехода, $k$ - постоянная Больцмана [6]. Выражение (2) может применяться для описания изотипных гетеропереходов, не имеющих тенденции к насышению прямой ветви ВАХ [6], что соответствует исследуемой структуре. В нашем случае выражение (2) описывает гетеропереход por-Si/p-Si. В диапазоне температур 8-300 K величина $n$ изменяется от 4.2 до 1.7 , следовательно, в этих условиях механизм токопрохождения определяется рекомбинацией носителей в области пространственного заряда гетероперехода por-Si $/ p-\mathrm{Si}$. Так как при $T=250-300 \mathrm{~K}$ участки 1 и 2 прямых ветвей BAX (рис. 2) практически сливаются, область действия указанного механизма токопрохождения распространяется на участок 1 в указанном температурном диапазоне.

Температурная зависимость плотности тока исследуемой структуры при $U=0$ В может быть представлена выражением

$$
J \propto \exp \left(\frac{-\Delta E}{k T}\right)
$$

где $\Delta E$ - высота барьера [6]. График, отражающий зависимость (3), приведен на рис. 4 в виде отрезка прямой в диапазоне температур $240-300 \mathrm{~K}$. Величина $\Delta E$, определенная по наклону графика, составила 0.55 эВ, что примерно соответствует половине ширины запрещенной зоны кремния. Следовательно, область пространственного заряда изотипного гетероперехода por-Si/p-Si преимущественно сосредоточена в $p$-Si. Экспериментальные точки при $T<240 \mathrm{~K}$ на графике (рис. 4) не показаны, так как в указанных условиях исследуемая структура имеет высокое сопротивление и плотность тока практически не зависит от температуры.

Таким образом, прямые ветви BAX на участке 2 при $T=80-300 \mathrm{~K}$, а также на участке 1 при $T=250-300 \mathrm{~K}$ могут быть описаны зависимостью

$$
J \propto \exp \left(\frac{-\Delta E}{k T}\right) \exp \left(\frac{q U}{n k T}\right),
$$

характерной для изотипного гетероперехода [6].

Участки 3 и 4 прямых ветвей $\mathrm{BAX}$ (рис. 2) при $T=80-300 \mathrm{~K}$ характеризуются разным наклоном, причем в обоих случаях $m>2$. В рамках модели ТОПЗ это может быть объяснено влиянием на процессы токопрохождения нескольких групп ловушек с разными значениями энергии активации [9].

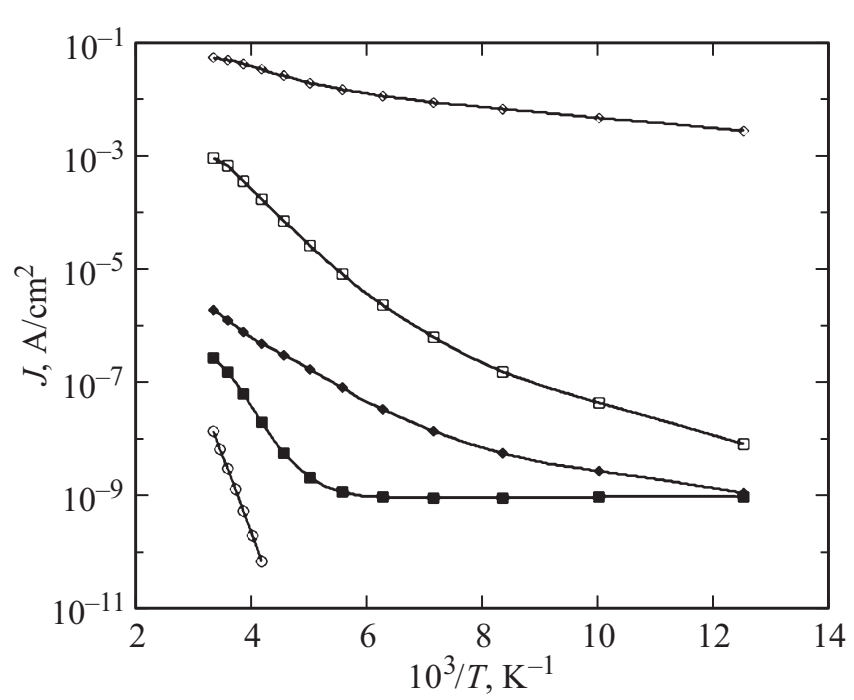

Рис. 4. Зависимость плотности тока от температуры при прямом смещении для значений $U, \mathrm{~B}: \circ-0, \square-0.6, \diamond-1.2$ и обратном смещении для значений $U, \mathrm{~B}: \mathbf{\square}-1.0,-6.0$. 


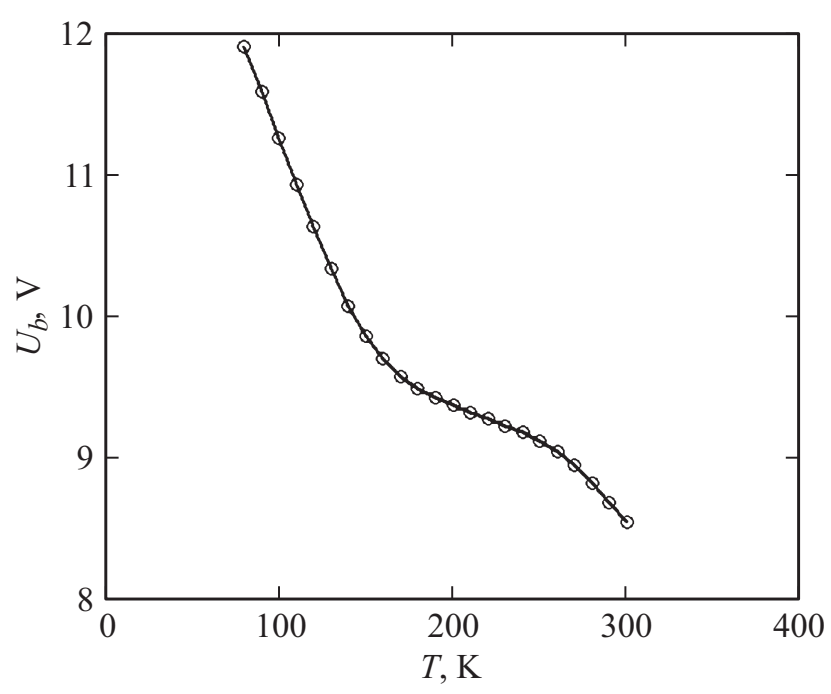

Рис. 5. Зависимость напряжения пробоя гетероструктуры $\mathrm{CdS} /$ por- $\mathrm{Si} / p$-Si от температуры.

Для прямых ветвей ВАХ при $T=80-140 \mathrm{~K}$ в области $U=1.0-1.8 \mathrm{~B}$, а также при $T=250-300 \mathrm{~K}$ и значениях $U=1.0-1.5$ В имеет место слабая зависимость величины $d(\ln J) / d V$ от температуры. Это свидетельствует о наличии туннельного механизма токопрохождения [6] на участке 5 и конечной области участка 4 прямых ветвей BAX (рис. 2) в указанных условиях. Туннелирование носителей заряда может происходить внутри пленки por-Si между энергетическими уровнями ловушек на поверхности кремниевых кристаллитов через барьеры $\mathrm{SiO}_{x}[10]$.

Графики температурной зависимости плотности тока для прямого смещения при $U>0$ В имеют вид гладких кривых (рис. 4). Это означает, что процессы токопрохождения определяются ловушками с энергиями активации, распределенными в некотором непрерывном диапазоне. Такая ситуация может быть характерна для поверхностных состояний. При $U=0.6 \mathrm{~B}$ энергия активации ловушек изменяется в пределах 0.059-0.190 эВ (рис. 4). При $U=1.2$ В диапазон энергии активации составляет 0.018-0.058 эВ. Наблюдаемое снижение энергии активации с ростом $U$ может быть объяснено особенностью пространственного распределения ловушек.

Ветви ВАХ при обратном смещении, измеренные в диапазоне температур 80-300 K, имеют достаточно четко выраженный участок пробоя (рис. 3). При $T=140-240 \mathrm{~K}$ и $U=5-7$ В имеет место наиболее слабая зависимость величины $d(\ln J) / d V$ от температуры. Согласно [6], это может свидетельствовать о преобладании туннелирования носителей заряда в указанных условиях.

Напряжение пробоя $\left(U_{b}\right)$ исследуемой структуры снижается с ростом температуры (рис. 5). Немонотонный характер кривой на рис. 5 может определяться конкуренцией двух процессов: наличием туннельной составляющей механизма пробоя и увеличением вклада генерации носителей заряда, связанной с перезарядкой энергетических уровней ловушек. Наблюдаемый рост наклона обратных ветвей ВАХ (рис. 3) в предпробойной области (0-6 В), особенно заметный в диапазоне температур 240-300 K, может быть объяснен возрастанием вклада генерационных процессов при перезарядке глубоких уровней ловушек.

Для области обратного смещения кривые температурной зависимости плотности тока представлены на рис. 4 для $U=1$ и $6 \mathrm{~B}$. При $U=1 \mathrm{~B}$ в области температур 190-300 K процессы токопрохождения определяются перезарядкой моноэнергетического уровня с энергией активации 0.282 эВ (рис. 4). В данном случае механизм токопрохождения можно также объяснить в рамках модели термоэлектронной эмиссии носителей заряда через потенциальный барьер для электронов [6] высотой 0.282 эВ, находящийся в зоне проводимости на гетерогранице por-Si $/ p$-Si со стороны $p$-Si. При $T<190 \mathrm{~K}$ и $U=1 \mathrm{~B}$ плотность тока практически не зависит от температуры, что может объясняться отсутствием влияния перезарядки энергетических уровней ловушек на процессы токопрохождения (рис. 4). При $U>3$ В в обратном смещении графики температурной зависимости плотности тока имеют вид гладких кривых, как и при прямом смещении. При $U=6 \mathrm{~B}$ энергия активации ловушек изменяется в пределах $0.031-0.148$ эВ (рис. 4). Наблюдаемое снижение энергии активации с ростом $U$ можно объяснить особенностью пространственного распределения ловушек.

\section{4. Выводы по результатам эксперимента}

Учитывая особенности процессов токопрохождения, установленные на основе анализа спектра фотоэдс (рис. 1), а также температурной зависимости ВАХ, измеренной в темновых условиях (рис. 2 и 3), можно предложить упрощенную схему зонной диаграммы исследуемой гетероструктуры $\mathrm{CdS} /$ por-Si/p-Si при равновесных условиях в виде рис. 6. Стрелками на рис. 6 условно показаны процессы генерации и разделения электронно-дырочных пар в гетеропереходе $p o r-\mathrm{Si} / p-\mathrm{Si}$ при освещении образца, а также процессы переноса созданных таким образом носителей заряда к внешним контактам. Параметры величин разрывов разрешенных энергетических зон являются предметом для проведения дальнейших исследований.

Прямые ветви ВАХ (рис. 2) на участке 1 при $T=250-300 \mathrm{~K}$ и участке 2 при $T=80-300 \mathrm{~K}$ могут быть описаны выражением (4) и определяются рекомбинацией носителей в области пространственного заряда изотипного гетероперехода por- $\mathrm{Si} / p$-Si, которая главным образом сосредоточена в $p$-Si. Зонная диаграмма изотипного гетероперехода на рис. 6 часто используется в литературе при описании свойств структуры por $-\mathrm{Si} / p-\mathrm{Si}$ (например, [10]). В пользу выбранной модели также 


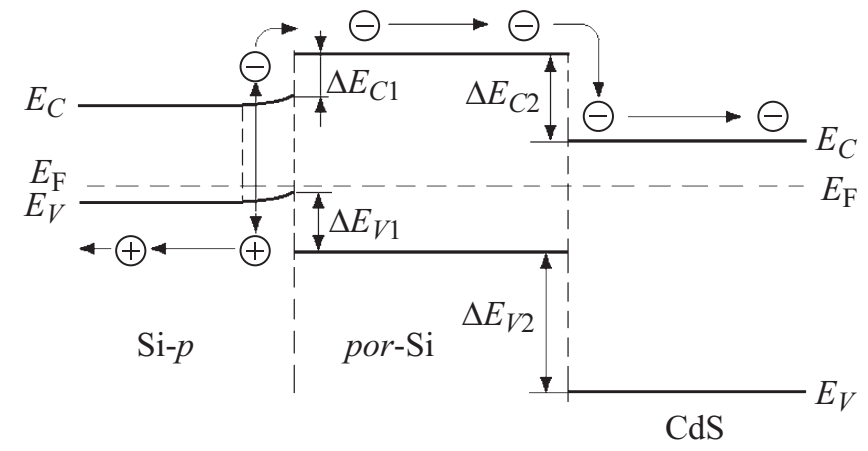

Рис. 6. Схема зонной диаграммы гетероструктуры $\mathrm{CdS} /$ por-Si $/$ p-Si.

свидетельствует тот факт, что режим прямого смещения этого гетероперехода возникает при подаче положительного потенциала на узкозонный полупроводник ( $p$-Si) [6], что соответствует исследуемому случаю.

При более высоких напряжениях прямого смещения (участки 3,4,5 на рис. 2) приложенное напряжение распределяется между por-Si и CdS. Здесь процессы токопрохождения определяются перезарядкой ловушек и объясняются в рамках модели ТОПЗ. Пленка $\mathrm{CdS}$ представляет собой частично компенсированный полупроводник $n$-типа проводимости, содержащий ловушки с мелкими и глубокими энергетическими уровнями, возникающими вследствие наличия вакансий серы и избыточного количества кадмия [11], а также сопутствующих химических примесей. Кроме того, в этой области прямых ветвей BAX (участки 4,5 на рис. 2) наблюдается туннелирование носителей заряда внутри пленки por-Si с участием ловушек. Вследствие высокой плотности поверхностных состояний на анизотипном гетеропереходе por-Si/CdS уровень Ферми может оказаться зафиксированным на гетерогранице [12]. При этом заметной модуляции ширины области пространственного заряда гетероперехода por-Si/CdS приложенным напряжением смещения наблюдаться не будет и изгибом зон можно пренебречь (рис. 6). По этой же причине уровень Ферми может быть зафиксирован на гетерогранице por-Si/p-Si со стороны por-Si, следовательно, изгибом зон в por-Si здесь также можно принебречь (рис. 6).

Моделью ТОПЗ можно объяснить токопрохождение на начальном участке прямых ветвей ВАХ при низких температурах (участок 1 на рис. 2 при $T=80-240 \mathrm{~K}$ ).

В обратном смещении процессы токопрохождения определяются туннелированием носителей в слое por $-\mathrm{Si}$ и генерацией в области пространственного заряда гетероперехода por-Si/p-Si, связанной с перезарядкой энергетических уровней ловушек.

При освещении исследуемой гетероструктуры кванты света проходят через слои $\mathrm{CdS}$ и por-Si, которые играют роль оптического окна, и поглощаются в $p$-Si. Электронно-дырочные пары разделяются полем гете- роперехода $p o r-\mathrm{Si} / p-\mathrm{Si}$ (рис. 6). Дырки выводятся из области пространственного заряда в квазинейтральную область $p$-Si и далее к внешнему контакту. Электроны проходят над барьером $\Delta E_{C 1}$ в por-Si, далее попадают в $\mathrm{CdS}$ и на внешний контакт. При этом часть электронов захватывается ловушками (эти процессы на рис. 6 не показаны). Фотогенерация носителей в $\mathrm{CdS}$ и por-Si возможна в коротковолновой области спектра. Однако эти процессы в нашем случае не вносят существенного вклада в общий фототок вследствие рекомбинации носителей с участием ловушек. В связи с этим наиболее вероятной причиной увеличения ширины области спектральной чувствительности гетероструктуры $\mathrm{CdS} /$ por- $\mathrm{Si} / p$ - $\mathrm{Si}$ по сравнению с фотоэлектрическим преобразователем на основе $n^{+}-p$-перехода (рис. 1) можно считать интерференционные эффекты в системе $\mathrm{CdS} /$ por-Si.

Применению гетероструктуры $\mathrm{CdS} /$ por $-\mathrm{Si} / p$-Si, исследуемой в данной работе, в качестве фотоэлектрического преобразователя солнечной энергии препятствует достаточно большое последовательное сопротивление, которое при $T=300 \mathrm{~K}$ составляет 40.2 Ом. Как известно, даже небольшой рост последовательного сопротивления существенно снижает эффективность фотоэлектрических преобразователей [12].

В то же время гетероструктура $\mathrm{CdS} /$ por $-\mathrm{Si} / p-\mathrm{Si}$ может быть использована для изготовления оптических датчиков с широкой полосой области спектральной чувствительности.

\section{5. Заключение}

Таким образом, процессы токопрохождения в исследуемой гетероструктуре $\mathrm{CdS} /$ por $-\mathrm{Si} / p$-Si определяются несколькими механизмами: генерационно-рекомбинационными процессами в области пространственного заряда гетероперехода por- $\mathrm{Si} / p$-Si, туннелированием и моделью ТОПЗ. Как в прямом, так и в обратном смещении, процессы токопрохождения определяются ловушками, энергии активации которых распределены в непрерывном диапазоне значений.

Гетероструктура $\mathrm{CdS} /$ por-Si/p-Si может быть использована при создании оптических датчиков с широкой полосой спектральной чувствительности. Проведение работ, направленных на оптимизацию технологии формирования слоев por-Si и $\mathrm{CdS}$, а также омических контактов с целью снижения последовательного сопротивления, позволит изготавливать на основе гетероструктуры CdS/ por-Si/ p-Si фотоэлектрические преобразователи солнечной энергии.

Представленные результаты получены в рамках выполнения государственного задания Министерства образования и науки России № 3.9506.2017/БЧ в Рязанском государственном университете им. С.А. Есенина, а также в рамках работ по гранту Президента Российской Федерации № 14.Z56.16.4518-МК в Рязанском государственном радиотехническом университете. 


\section{Список литературы}

[1] Handbook of Porous Silicon, ed. by L. Canham. (Springer International Publishing, 2014).

[2] J.P. Zheng, K.L. Jiao, W.P. Shen. Appl. Phys. Lett., 61, 459 (1992).

[3] H.A. Thjeel, A.M. Suhail, A.N. Naji. Adv. Mater. Phys. and Chem., 1, 70 (2011).

[4] S.A. Hasoon, I.M. Ibrahim, M.S. Raad. Int. J. Current Engin. Technol., 4 (2), 594 (2014).

[5] M.A. Jafarov, E.F. Nasirov, S.A. Jahangirova. Int. J. Sci. Engin. Research, 6 (7), 849 (2015).

[6] Б.Л. Шарма. Полупроводниковые гетеропереходы (М., Сов. радио, 1979).

[7] L. Fangyang, L. Yanging, L. Jun. J. Alloys Comp., 493 (1, 2), 305 (2010).

[8] Ю.Б. Болховитянов, О.П. Пчеляков, С.И. Чикичев. УФН, $171(7), 689$ (2001).

[9] М. Ламперт, П. Марк. Инжекционные токи в твердых meлаx (М., Мир, 1973).

[10] А.А. Евтух, Э.Б. Каганович, Э.Г. Манойлов, Н.А. Семененко. ФТП, 40 (2), 180 (2006).

[11] К. Чопра, С. Дас. Тонкопленочные солнечные элементы (М., Мир, 1986).

[12] А. Фаренбух, Р. Бьюб. Солнечные элементы: Теория $и$ эксперимент (М., Энергоатомиздат, 1987).

Редактор Г.А. Оганесян

\section{Investigation of Currrent Flow Mechanisms in the $\mathrm{CdS} / p o r-\mathrm{Si} / p-\mathrm{Si}$ heterostructure}

\section{V.V. Tregulov1, V.G. Litvinov², A.V. Ermachikhin ${ }^{2}$}

${ }^{1}$ Ryazan State University named for $S$. Yesenin, 390000 Ryazan, Russia

${ }^{2}$ Ryazan State Radio Engineering University, 390005 Ryazan, Russia

Abstract The temperature dependence of the forward and reverse bias of the current-voltage characteristic, as well as the spectrum of the photo-emf of the semiconductor heterostructure $\mathrm{Cds} /$ por-Si $/ p$-Si, are studied. It is found that the current flow mechanisms determined by generation-recombination processes in the space charge region of a geterojunction por-Si $/ p$-Si, tunneling of charge carriers in por-Si film and a model of the currents limited by the space charge. A cimplified version of the band diagram of the investigated heterostructure has been proposed. 